PALAVRAS. Revista de Epistemología, Metodología y Ética del Psicoanálisis

ISSN: 2468-9831

www.revistas.unlp.edu.ar/palavras

palavras@outlook.com.ar

Argentina

\title{
LA TRADICIÓN ASCÉTICA Y EPISTÉMICA EN LA PSICOLOGÍA Y EL PSICOANÁLISIS
}

DOI $10.24215 / 24689831 \mathrm{e} 024$

Jennifer Ortiz Vanegas

\begin{abstract}
This article deals with the psychology's state and its different branches, conceived both as a product of two ancient approaches to the psyche: an ascetic one privileging the treatment and care of the psyche and an epistemic one inquiring into a systematic knowledge of the psyche. Several epistemological consequences for the field of psychology are derived from these two approaches, regarding its object of study and its relation to psychoanalysis. This analysis leads to conclude that not all branches of psychology have been concerned with becoming a science and that philosophy and spirituality are not only predecessors of psychology, but they constitute a field in contemporary psychology.
\end{abstract}

Key-words: ascesis - episteme - psychology psychoanalysis - psyche.
Resumen

Este artículo permite comprender el estado actual de la psicología y sus diferentes escuelas como producto de dos tradiciones antiguas en el abordaje del alma, una ascética, que privilegia el tratamiento y cuidado del alma, y otra epistémica, que privilegia la investigación para obtener un conocimiento sistemático sobre el alma. De alli se derivan varias consecuencias epistemológicas para esta disciplina respecto a su objeto de estudio y a su relación con el psicoanálisis; se evidencia que no todas las escuelas psicológicas se han constituido con el propósito de ser ciencia y que la filosofia y la espiritualidad no son solamente antecedentes de la psicología y parte de su pasado remoto, sino que constituyen una vertiente presente en la psicología contemporánea.

Palabras clave: ascesis - episteme - psicología psicoanálisis - alma

\section{Cómo citar este artículo:}

Ortiz Vanegas, J. (2019).La tradición ascética y epistémica en la psicologia y el psicoanálisis. Palavras. Revista de Epistemología, Metodología y Ética del Psicoanálisis, 4, 52-69. Recuperado de www.revistas.unlp.edu.ar/palavras 


\section{LA TRADICIÓN ASCÉTICA Y EPISTÉMICA EN LA PSICOLOGÍA Y EL PSICOANÁLISIS}

\section{Jennifer Ortiz Vanegas*}

\section{Introducción}

Que el psicoanálisis forma parte de la psicología o es una escuela psicológica, como lo es el conductismo, es una afirmación que dista mucho de ser obvia y sería dificilmente aceptada por psicoanalistas y psicólogos, por lo menos en el contexto colombiano. En este contexto las relaciones entre la psicología y el psicoanálisis han sido complejas, predominando una posición que establece una diferencia y exclusión radical entre ambos saberes.

Los psicoanalistas asumen la psicología como una disciplina con un corpus unificado que se estableció en la modernidad con el propósito de constituirse como ciencia, es decir, asumen que psicología es sólo la psicología clásica, también llamada académica, experimental o de corte cognitivo-conductual, mientras que el psicoanálisis es un discurso y una práctica que - para Lacan, no para Freud-se opone radicalmente a la ciencia. Quienes piensan de este modo, por un lado, ignoran o consideran un equivoco de Freud su proyecto de que el psicoanálisis estuviera lo más cerca posible del discurso científico; y, por otro lado, desconocen que también forman parte de la psicología otras escuelas, como la dinámica, la humanista y la transpersonal, que se han constituido con propósitos muy distintos a enmarcarse en el campo de la ciencia.

Algunos psicólogos, por su parte, no reconocen la formación positivista de Freud como médico neurólogo, los criterios de validación científica que utilizaba en la aplicación del método psicoanalítico, sus intentos de objetivar y generalizar, tal como pretende la ciencia, sus teorias, hasta donde la honestidad científica se lo permitia, su expectativa de que en el futuro el avance de la ciencia permitiera abordar desde una perspectiva biológica $\mathrm{y}$

\footnotetext{
*Universidad de Antioquia, Colombia. Mail: jennifer.vanegas@gmail.com
} 
completamente objetiva lo que él sólo podía conjeturar desde una perspectiva psicológica; de hecho, en la actualidad ese es el proyecto de muchos de sus colegas neurólogos en el campo de investigación que se ha denominado neuropsicoanálisis.

Las diferencias entre psicólogos y psicoanalistas se han exacerbado en Colombia a propósito de las reformas curriculares de los programas de psicologia en años recientes, asunto que actualiza la pregunta por las relaciones entre estos dos saberes, la pertinencia de enseñar psicoanálisis en la universidad, específicamente en los programas de psicología, que cada vez más toman una orientación exclusivamente cognitivo-conductual y experimental, orientación que en muchas ocasiones se asume, lo hemos dicho, como la única y verdadera psicología.

A pesar de este contexto, marcadamente cientificista, están en auge propuestas psicológicas y psicoterapéuticas que recogen las inquietudes ascéticas, espirituales que la psicología científicamente respaldada ha dejado de lado. El propósito de este artículo es retomar algunos aspectos de los antecedentes y la historia de la psicología con el fin de comprender la diversidad de escuelas y propósitos que hay en el campo de la psicología y su relación con el psicoanálisis.

\section{Dos perspectivas en el abordaje del alma}

Desde la filosofia antigua podemos reconocer y diferenciar dos tendencias en el abordaje del alma (Psyche), una que llamaremos ascética y otra epistémica. Lo que caracteriza la tendencia ascética es que privilegia la pregunta por la transformación del alma, el logro de la virtud o excelencia del ser humano (Areté) indispensable para acceder a la verdad. Mientras que la tendencia epistémica privilegia la construcción de un conocimiento sistemático sobre el alma, sobre su origen, su naturaleza y sus facultades. El interés que entrañan estas dos tradiciones para la psicología es que constituyen el punto de partida y fundamento de las psicologias epistémicas y ascéticas. En una aproximación bastante esquemática miremos en qué consiste cada una de ellas y su devenir. 
Cuando Michel Foucault hace un estudio de la relación del sujeto con la verdad, destaca el lugar que en esa relación tiene la epimeleia heautou, un concepto que recorre todo el pensamiento griego y puede entenderse como inquietud de sí mismo (Foucault, 2006), una actitud que consiste en ocuparse de sí, cuidar de sí e incluye todas aquellas acciones que el sujeto realiza sobre sí mismo y a través de las cuales se modifica, se purifica, se transforma y se transfigura, y es de este modo que puede acceder a la verdad, ya que el acceso a la verdad coincidía con la adquisición de la virtud, que según Platón era propia de los verdaderos filósofos, quienes se ocupaban exclusivamente del cultivo de su propio alma (Platón, 2005), de allí el interés de la epimeleia heautou para los filósofos.

Destaca Foucault que esa era la función esencial de Sócrates, "incitar a los otros a ocuparse de sí mismos, a cuidar de sí mismos y no ignorarse" (2006, p. 20). En el Protágoras, por ejemplo, Sócrates cuestiona a Hipócrates porque quiere volverse aprendiz de Protágoras sin saber muy bien a qué clase de hombre le estaría confiando su alma para que la cuide, sin saber a qué peligros la expone al dejarla en manos de un sofista (Platón, 1998). Vemos entonces la implicación que tiene para Sócrates elegir un maestro: es elegir una persona a quien se le otorga el cuidado del alma, el poder de trans-formarla, de orientar, según sus valores, ese proceso de ascesis subjetiva; por eso le dice a Hipócrates que debe tener mucho cuidado con las enseñanzas que está dispuesto a comprar, pues es de ellas que se nutre el alma, así como de alimentos se nutre el cuerpo. Y en su apología, Sócrates afirma que con su muerte pierden más los atenienses al quedarse sin alguien que los exhorta sin cesar a preocuparse por sí mismos, a cuidar de su alma, de la verdad y de la razón, más que de la fortuna, la reputación y los honores (Platón, 2010).

Los estoicos, los epicúreos y los cínicos privilegiaron más aún la construcción y difusión de un saber sobre el talante espiritual y las prácticas necesarias para vivir de la mejor manera posible en medio del sufrimiento que entraña la vida; más que la búsqueda de la verdad y la construcción de una episteme 
(conocimiento sistemático) sobre el alma, este era el principal sentido de la filosofia para ellos (Abbagnano, 1974; Lopera, 2006). Sobre estas escuelas filosóficas plantea María Zambrano:

Porque estos filósofos, cínicos, y sobre todo estoicos y epicúreos, eran filósofos para ser en realidad otra cosa, una especie de médicos, de curas párrocos o frailes caritativos. Habían acudido a la razón, sí, mas no a la razón perfecta y desarrollada, la platónico-aristotélica, sino a alguna otra anterior a ella, más reducida y más convincente. Con el nombre de filósofos vivian algo muy parecido a una religión (Zambrano, 1987, p. 20).

También en la Antigüedad interesaba el alma como objeto de indagación, por supuesto, y quizá el mayor exponente de la tendencia epistémica, en este periodo, lo hallamos en "aquel para quien, entre los filósofos, tuvo menos importancia la cuestión de la espiritualidad" (Foucault, 2006, p. 35). De los filósofos clásicos Aristóteles fue el que más privilegió la episteme, producto de ello su tratado Acerca del alma, donde indaga y conceptualiza este asunto de manera sistemática.

La actitud o ejercicio filosófico griego de ocuparse de sí mismo evoluciona en la Edad Media hacia el ascetismo cristiano, cuyas prácticas estaban relacionadas con la restricción de los placeres (el celibato, el ayuno, el sacrificio, la renuncia, el castigo y la mortificación del cuerpo) para lograr la purificación del alma y conducirla nuevamente a Dios. Entonces, mientras que el ascetismo griego implica un cuidado de sí, ocuparse de sí para acceder a la verdad, el ascetismo cristiano implica rechazar una parte de sí para volver a Dios, ubicado en el lugar de la verdad.

En Occidente se ha denominado Edad Media a un periodo que en realidad es específico de Europa - China, el imperio persa, el imperio otomano, por ejemplo, viven en este periodo una historia distinta, aunque por la hegemonía que ha tenido la versión europea de la historia se suele pensar que describe el contexto de toda la humanidad en esa época- caracterizado por un espíritu religioso predominante, que bajo el poder de la Inquisición promovía la creencia ciega en los dogmas y doctrinas de la Iglesia Católica. Este ambiente hostil al 
escepticismo truncó por mucho tiempo el desarrollo de investigaciones y la divulgación de nuevos hallazgos. Los medios para difundir el conocimiento y el poder de validarlo estaban en manos de los escolásticos. En este contexto medieval se le da más relevancia al ascetismo, por su función moralizante, que a la tendencia epistémica, siempre en riesgo de contradecir los dogmas de la iglesia. Esto va generando una separación cada vez más marcada entre las dos tradiciones, disociación que llega a su culmen en la Modernidad, como veremos a continuación.

Del interés incesante de los filósofos por hallar un método que conduzca a la verdad surge la propuesta cartesiana de la duda metódica, que implicaba poner en cuestión todos los conocimientos escolásticos para hacer una revisión exhaustiva de ellos (Descartes, 1970), y la propuesta del empirismo inglés que enfatiza la importancia de la observación de los hechos para acceder al conocimiento verdadero (Bacon, 1984). Estas dos propuestas, articuladas, van a configurar el método científico utilizado por Galileo y Newton, y anteceden el surgimiento de la ciencia moderna en el siglo XVII.

El prestigio que adquirieron las teorias científicas de la física, la astronomía, la química, la óptica, generó gran optimismo hacia esta nueva forma de obtener el conocimiento, al punto de creer que con la utilización del método científico se llegaría a conocer completamente la realidad física, social y psíquica (Cassirer, 1981). Así como la química había logrado diferenciarse de la alquimia y la astronomía de la astrología, en todas las áreas del conocimiento surgió el interés de adherir a la ciencia y dejar atrás las teorias metafisicas y filosóficas, consideradas inútiles a la luz de los nuevos hallazgos.

Sin embargo, abordar el alma con el método experimental implicaba responder de manera precisa acuciantes preguntas sobre este objeto de estudio: ¿qué es el alma?, ¿cuál es su naturaleza?, ¿'es material o inmaterial?, ¿es viable observarla y medirla para someterla a experimentación? Ante la evidente dificultad que entrañaba la matematización y control experimental del alma, surgieron propuestas de cambiar el objeto de estudio, no la Psyche, sino las manifestaciones psíquicas susceptibles de ser abordadas con el método 
experimental (la sensación, la percepción, la atención, la memoria, las emociones), y así fundar la psicología como una ciencia natural. De este corte epistémico son las propuestas de Wilhelm Wundt y John Watson. El primero se opone a concebir la psicología como el estudio de nebulosos asuntos como el alma o la experiencia interna, y en su lugar propone el estudio experimental de la experiencia inmediata (Wundt, 1982). El segundo propone el estudio de la conducta observable como objeto de la psicología y fundarla como una rama experimental puramente objetiva de la ciencia natural (Watson, 1982). Al proponer un objeto observable pretende evitar el recurso a la subjetiva introspección, un escollo que no pudo evitar Wundt.

Este contexto de la Modernidad es llamado por Foucault el momento cartesiano, y plantea como hipótesis que es el asunto de la verdad lo que está en juego y por lo cual queda descalificada la tradición ascética y excluida del campo del pensamiento filosófico moderno en esta época (Foucault, 2006, p. 32-33), ya que la forma de proceder cartesiana sitúa las evidencias en el punto de partida de una indagación válida. Descartes marca un rumbo trazado por las evidencias, tan apreciadas por el positivismo.

En el campo de la espiritualidad se postula que el sujeto no accede a la verdad en un mero acto de conocimiento, es preciso que se modifique, se transforme, se desplace, se convierta en algo distinto de sí mismo, que haga un movimiento de ascensión que lo saque de su condición actual — se vuelva virtuoso o alcance cierta excelsitud, dirían los filósofos clásicos- para llegar a ser, por fin, un sujeto capaz de acceder a la verdad, una verdad que tiene el efecto de iluminarlo, de darle tranquilidad a su alma, de pacificarlo. Por tanto, el acceso a la verdad en la tradición ascética implica un trabajo sobre sí mismo, una transformación de sí mismo de la que uno es responsable, "una prolongada labor que es la de la ascesis" (Foucault, 2006, p. 34). Cuando el asunto de la verdad entra en el periodo moderno se admite, en cambio, que es el conocimiento mismo y por sí solo lo que da acceso a la verdad.

En la Modernidad acceder a la verdad ya no tiene condiciones espirituales (ascéticas), sino formales, objetivas, reglas formales del método de investigación 
y condiciones estructurales del objeto a conocer; una serie de condiciones de formalidad en el conocimiento, no en el sujeto. Mientras que en la Antigüedad no es el conocimiento, sino la transformación lo que le permite al sujeto acceder a la verdad.

Este conocimiento formal de la modernidad no es la verdad a la que puede acceder el sujeto cuando vive un proceso espiritual de transformación subjetiva, es un conocimiento objetivo, válido para la ciencia, pero desprovisto del poder de iluminarlo, de pacificarlo. Podemos sintetizar estas ideas con algunas fórmulas foucaultianas:

- En la Antigüedad el sujeto, tal como es, no tiene las condiciones para acceder a la verdad, pero esta, tal como es, tiene el poder de transformarlo. En la Modernidad el sujeto, tal como es, puede acceder a la verdad, pero esta, tal como es, no tiene el poder de transformarlo.

- En la Antigüedad la verdad pasa por la espiritualidad, por la transformación subjetiva (ascesis); en la Modernidad la verdad pasa por el conocimiento (episteme).

Esta es la conjetura foucaultiana a partir de la cual se explicaría que en la modernidad hayan sido menos populares las propuestas de constituir la psicologia conservando sus raíces ascéticas, y que, en lugar de cambiar el objeto para que se ajustara al método experimental, la psicología creara sus propios métodos de investigación, acordes con la realidad que estudia. Un ejemplo lo hallamos en la propuesta de Wilhelm Dilthey, quien considera que la aplicación del método experimental de las ciencias naturales y los intentos de explicación serian infructuosos en la psicologia, porque la naturaleza de su objeto, la vida psíquica, no da la posibilidad de descartar hipótesis. En lugar de una psicología explicativa propone una psicología comprensiva, cuyo propósito sea describir la estructura y regularidades de la vida psíquica, y para ello utiliza la hermenéutica como método de investigación propio de las ciencias del espíritu (Dilthey, 1945).

Se hace evidente entonces el precio que debe pagar la psicología por acceder al conocimiento bajo las condiciones formales que implica la ciencia: renunciar 
a la tradición ascética en el abordaje del alma, a sus raíces filosóficas y espirituales, porque el conocimiento objetivo de la ciencia no logra nombrar ni reemplazar el saber que adquiere el sujeto en el proceso de transformación por el cual accede a su verdad más entrañable y se hace excelso. La episteme, el saber formalizado y sistemático, no abarca todo el campo de la experiencia humana y, en particular, no hay una episteme de aquello que realiza la perfección, la areté de esa experiencia (Lacan, 1983). Martha Nussbaum retoma a Aristóteles cuando plantea la imposibilidad de que un saber formalizado, como la ciencia, logre nombrar y transmitir la sabiduría práctica, que se adquiere en el hacer, en la experiencia:

Aristóteles insiste [...] en que la sabiduría práctica no es episteme, o sea, comprensión científica sistemática. Defiende su posición argumentando que tiene que ver con particulares últimos (takath' hekasta) que no pueden ser incluidos en ninguna episteme (sistema de principios universales) sino que tienen que ser aprehendidos por medio de la experiencia (Nussbaum, 1995, p. 123).

La siguiente gráfica ilustra la tendencia predominante en cada época, ubicando por encima de la línea del tiempo aquella que fue privilegiada, y por debajo aquella que fue eclipsada, sin que ello implique su desaparición, pues ambas han coexistido a lo largo de la historia.

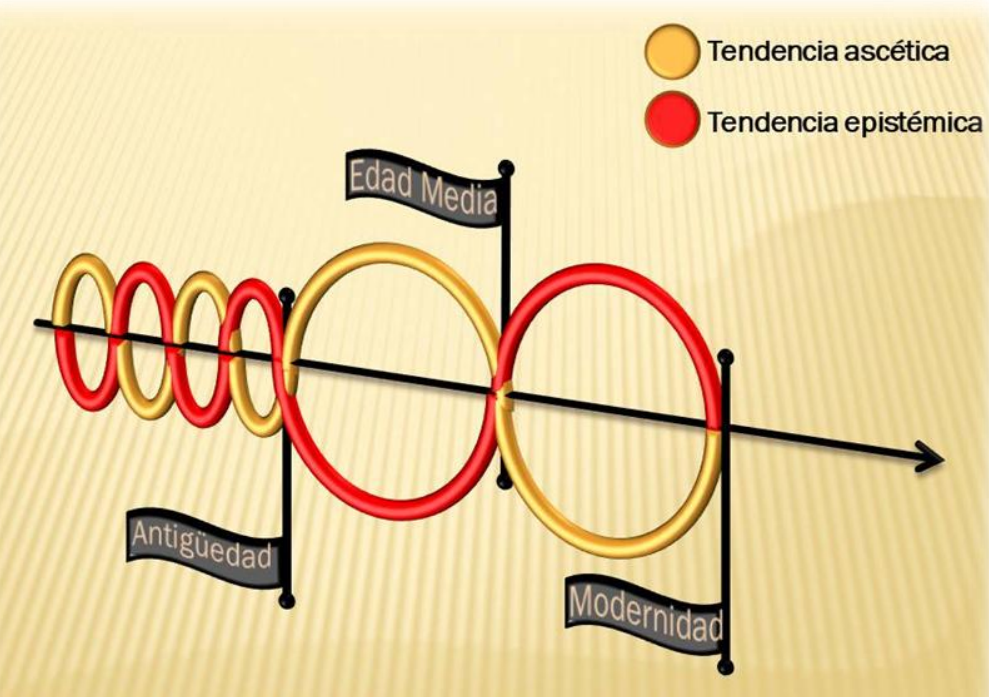


En la Antigüedad predomina la dialéctica de ambas tendencias. Foucault destaca que en la Antigüedad el conocimiento filosófico y el ascetismo, la espiritualidad, nunca se separaron. Estas dos tendencias estaban imbricadas. En la Edad Media, en cambio, es predominante el interés ascético en su versión religiosa. Situación que se invierte en la modernidad, cuando la ciencia moderna devino un paradigma para todas las áreas del conocimiento.

Herederas de la tradición epistémica en psicología son la escuela cognitivoconductual, la neuropsicología, la psicología experimental y la psicología evolucionista, que han privilegiado el propósito de producir conocimiento validado por la comunidad científica, por lo que intentan ajustarse lo más que se pueda a los criterios de universalidad, objetividad, univocidad y matematización que exige la ciencia moderna. Herederas de la tradición ascética consideramos al psicoanálisis, la psicología dinámica o relacional, la escuela humanista y la psicologia transpersonal por el valor que le otorgan a la transformación subjetiva a través de la psicoterapia y la práctica clínica, experiencias modernas y contemporáneas de ascesis subjetiva, escuelas que no han privilegiado la construcción sistemática de teorías que hagan parte de la ciencia.

Queda por asentar un eslabón en esta elaboración, y es que venimos dando por supuesto el alma como objeto de estudio de la psicología, asunto que amerita ser aclarado. Hemos dicho que desde la Antigüedad el alma se ha abordado desde dos perspectivas, una ascética y otra epistémica, y que ambas tradiciones persisten en la psicología actualmente. El lector se preguntará qué tiene que verla psicologia contemporánea con el abordaje ascético o epistémico del alma (Psyche) en la filosofia antigua, pues comúnmente se asume y se da por hecho que el alma dejó de ser el objeto de estudio de la psicología y, más aún, que cada escuela psicológica se ocupa de un objeto de estudio diferente. Creemos que son presupuestos equivocados.

Si definimos el alma, no como una entidad metafisica que preexiste al organismo y lo trasciende, sino como la cultura encarnada en un organismo humano, es posible afirmar que todas las escuelas psicológicas, ascéticas y 
epistémicas, se ocupan de este objeto de estudio (Lopera, Manrique, Zuluaga, \& Ortiz, 2010; Ramírez, 2012). Vale la pena aclarar un poco más esta idea.

Entendemos el alma como una estructura que emerge en el ser humano cuando éste ingresa a la cultura por medio de un proceso de formación, individuación, humanización, crianza, educación, culturización o sujetación. Como resultado de este proceso que vive el ser humano, decimos, emerge una estructura psíquica, que es estudiada por todas las escuelas psicológicas aunque la nombren de manera distinta: aparato psíquico, mente o procesos psíquicos, personalidad, identidad, subjetividad... Formas distintas de nombrar el alma humana, conceptos que se han propuesto, entre otras cosas, para evitar la palabra alma o Psyche, por las resonancias religiosas y metafísicas que tiene. Tanto es así que desde las escuelas conductistas se ha propuesto abandonar la palabra psicología y hablar de ciencia de la conducta o del comportamiento. Sin embargo, consideramos que la psicología no solamente estudia la conducta o el comportamiento, estos serían los efectos o resultados de la estructura psíquica y la manera particular en que se dio el proceso de estructuración en cada ser humano. Una psicología que deje de lado el estudio de la estructura psíquica, de la que emergen las conductas, sería una psicología cercenada, incompleta.

Este objeto de estudio, común a todas las escuelas de psicologia, puede abordarse desde ambas perspectivas y con propósitos distintos. Desde una perspectiva epistémica, con todo el rigor de los métodos científicos y con el propósito de dilucidar teorias sistemáticas acerca de su constitución, estructura, funcionamiento y efectos; o puede abordarse desde una perspectiva ascética, con el propósito, ya no de elaborar un tratado, sino un tratamiento del alma (psicoterapia), lo que hemos denominado procesos de ascesis subjetiva relacionados con el campo de la espiritualidad.

\section{¿Es el psicoanálisis una psicologia?}


El debate surgido en la modernidad, si la psicología debía constituirse como ciencia, renunciando para ello a la tradición ascética, subyace también a la pregunta de si el psicoanálisis hace parte de la psicología o no. Quizá la respuesta se considere obvia, el problema es que algunos consideran que la respuesta obvia es que no y otros que sí. Para aportar una respuesta epistemológicamente rigurosa con ambas disciplinas, será preciso definir muy bien qué entendemos por psicología y cómo definimos el psicoanálisis.

Tomemos como punto de partida la definición etimológica de psicología como estudio, tratado, discurso, conocimiento acerca del alma, concepto que previamente definimos como cultura encarnada, definición tan laica como antigua, ya que puede remontarse hasta la propuesta del hilemorfismo en Aristóteles (Aristóteles, 1983), pero perfectamente compatible y abordable, ya lo hemos dicho, con los métodos de la ciencia moderna (cf. Lopera et al., 2010; Ramirez, 2012).

El psicoanálisis [en palabras de Freud] es el nombre: 1) de un procedimiento que sirve para indagar procesos anímicos dificilmente accesibles por otras vías, 2) de un método de tratamiento de perturbaciones neuróticas, fundado en esa indagación, y 3) de una serie de intelecciones psicológicas, ganadas por ese camino, que poco a poco se han ido coligando en una nueva disciplina científica (Freud, 1923b, p. 231).

Y en su ensayo El provenir de una ilusión plantea que "el psicoanálisis es un método de investigación, un instrumento neutral, como lo es por ejemplo, el cálculo infinitesimal" (Freud, 1927, p. 36).

Tenemos entonces que el psicoanálisis es, esencialmente, un método, que puede aplicarse para investigar procesos anímicos (propósito epistémico) o para tratar la neurosis (propósito ascético) en el dispositivo freudiano. Y como resultado de la aplicación del método psicoanalítico se obtienen teorías, algunas de ellas sobre metodología y técnica psicoanalítica, otras son teorias antropológicas, sociológicas, neurológicas. Pero aquellas teorias psicoanalíticas referidas específicamente al aparato psíquico, a sus etapas de conformación, sus instancias, mecanismos, economía, dinámica, sus efectos (sueños, chistes, 
lapsus, sintomas), etc., estas teorias, llamadas por Freud metapsicológicas, hacen parte de la psicología, ya que son un conocimiento, un tratado sobre el alma o, como decía Freud, sobre el aparato psíquico, objeto de estudio de la psicología (Ramírez et al., 2014).

A diferencia de los psicoanalistas contemporáneos, Freud reconocía que sus teorias eran psicológicas y hacian parte de esta disciplina: "el psicoanálisis deberá calificarse como una psicología del Ello (y de sus acciones eficaces sobre el Yo)" (Freud, 1923a, p. 220). Néstor Braunstein, por su parte, afirma que estas teorias constituyen la base de una psicología psicoanalítica (Braunstein, 1979).

Para clarificar más esta afirmación es preciso enfatizar la diferencia entre método científico y ciencia, distinción que permite sustentar, incluso, que el psicoanalítico es un método científico, afirmación que puede producir rechazo en los claustros de psicología experimental, por eso es importante sustentarla.

La ciencia (el conocimiento) de la Antigüedad debía pasar por el filtro de la lógica, mientras que la ciencia de la modernidad implica que las teorías no sólo sean lógicas, sino que deben ser corroboradas por la experiencia, es por ello que el método experimental se toma como paradigma del método científico, a tal punto que suele creerse que son equivalentes, desconociendo otros métodos también científicos aunque no experimentales. Veamos.

Lo que define que un método sea científico es que lleve a cabo una contrastación dialéctica entre la teoría y la práctica (Ramírez, 1991), y el método experimental - también llamado hipotético deductivo o galileanonewtoniano- es sólo una manera de llevar a cabo esta contrastación; otros métodos como el clínico, el psicoanalítico o el de los críticos de arte, también proceden contrastando la teoría y la práctica, en un diálogo donde ambas se van modificando, y es esto lo que produce la transformación y el avance de la ciencia. Estos métodos no experimentales hacen parte del que Carlo Ginzburg (1989) denomina "paradigma indiciario", un paradigma científico alternativo al experimental. 
Los criterios de validación que utiliza la ciencia son la consistencia y la eficacia. El primer criterio es de orden teórico, evalúa la coherencia de las hipótesis, que no presenten contradicciones con la teoría de la cual derivan. El segundo es de orden práctico, y verifica si los efectos que se producen en la experiencia validan o invalidan las hipótesis (Ramírez, 1991). Freud plantea que el progreso en el trabajo científico se consuma exactamente como en un análisis (Freud, 1932), utilizando los criterios de la ciencia para verificar la validez de las interpretaciones (hipótesis). En la tercera conferencia de introducción al psicoanálisis plantea que para saber si una interpretación es correcta debe ser coherente con el discurso del paciente, con su historia y sus circunstancias personales (consistencia), y producir efectos que hagan avanzar el análisis (eficacia), generando sueños, asociaciones, recuerdos, formaciones de lo inconsciente y comportamientos que confirmen o invaliden la interpretación. Y rechaza como criterios de validez de una interpretación la aceptación del paciente, considerarla brillante o evidente (Freud, 1916).

Porque el método psicoanalítico procede contrastando dialécticamente la teoría y la práctica, y porque utiliza la consistencia y la eficacia como criterios de validación planteamos que es un método científico. Esto no implica que el psicoanálisis sea una ciencia; Freud nunca confundió el método con la ciencia, pues esta se refiere exclusivamente a los conocimientos que resultan de la aplicación del método científico y que, además, cumplen con los criterios que establece la comunidad científica de la época, en el caso de la modernidad estos criterios son: objetividad, universalidad, univocidad, matematización, falsabilidad, entre otros. El psicoanálisis no es una ciencia porque sus teorías, aunque derivan de un método científico, no cumplen con estos criterios (Ramírez, 1991).

Hasta ahora nos hemos referido al psicoanálisis para decir que su método es científico y su teoría, referida al aparato psíquico, hace parte de la psicología, mas no de la ciencia. Sólo queda por retomar el psicoanálisis en tanto tratamiento para la neurosis, el dispositivo freudiano. Sobre esta práctica clínica diremos que es lo que inscribe al psicoanálisis en la tradición ascética, 
porque consiste en una práctica que el sujeto efectúa sobre sí mismo y a partir de la cual puede obtener una verdad que le concierne exclusivamente a él, y el efecto que tiene esta verdad es la transformación subjetiva (reposicionamiento frente a la vida, al amor, la moderación del sufrimiento, de la angustia, de los sintomas).

Y como es característico de las prácticas ascéticas, gran parte del saber que se adquiere en un análisis no es transmisible como episteme, como conocimiento. Al no ser susceptible de objetivación, universalización y formalización no pasa por la evidencia de la ciencia. Al respecto dice Lacan que de lo que no puede ser demostrado tal como exige la ciencia, un conocimiento basado en evidencias, algo verdadero, empero, puede ser dicho (Lacan, 1982).

\section{Conclusiones}

1. Desde la filosofia clásica pueden rastrearse dos propósitos en el abordaje del alma, uno ascético, que busca la transformación del alma por medio de prácticas espirituales, de cuidado de sí; y otro epistémico, que busca estudiar el alma y construir un conocimiento sistemático sobre ella. En la Edad Media, gracias al discurso religioso predominante, se privilegió el abordaje ascético del alma. Mientras que en la Modernidad, por el auge y prestigio que adquirió la ciencia moderna, se buscó insistentemente fundar la psicología como ciencia y obtener conocimientos sistemáticos sobre el alma o sus manifestaciones (procesos psíquicos y conductas) tan contundentes como la física los obtenía de la naturaleza, aunque esto implicara excluir el abordaje ascético y la espiritualidad. Pero no todas las escuelas de psicología han privilegiado el proyecto de constituirse como ciencia siguiendo el paradigma de la modernidad, ni han pretendido que sus teorias se ajusten a los requerimientos de la comunidad científica, pues han dado más valor a aspectos ascéticos, espirituales, no susceptibles a un abordaje experimental ni a la sistematización, objetivación, matematización y formalización que exige la ciencia. Ambos propósitos continúan presentes en la psicología contemporánea y en torno a ellos se constituyen las 
psicologías ascéticas (psicoanalítica, dinámica, humanista y transpersonal) y epistémicas (conductismo, psicología cognitiva, evolucionista, experimental y neuropsicologia) respectivamente.

2. Visibilizar estas dos tradiciones permite reconocer que las psicologias ascéticas y epistémicas no se ocupan de objetos de estudio diferentes como habitualmente se cree. Al contrario, son dos maneras distintas de abordar un mismo objeto de estudio, el alma, entendida, no ya como una entidad metafísica que preexiste y trasciende el cuerpo, sino como la forma de ser (estructura) adquirida por un organismo humano cuando ingresa a la cultura. Cada una de las escuelas psicológicas nombra de manera distinta esta estructura o privilegia el estudio de algún aspecto de ella: aparato psíquico, personalidad, identidad, procesos psíquicos, mente, individuo, subjetividad. Las diferencias radican entonces en los métodos y propósitos al abordar el objeto y en la forma de conceptualizarlo, no en el objeto en sí.

3. Partiendo de la definición que Freud aporta del psicoanálisis, como el nombre que le da a un método de investigación y a las elaboraciones teóricas que resultan de la aplicación de dicho método, podemos hacer las siguientes afirmaciones: aquellas teorias psicoanaliticas referidas al aparato psíquico hacen parte de la psicología, constituyen la psicología psicoanalítica, pues son una contribución teórica sobre el objeto de estudio de la psicología. Es por el afán de algunas escuelas psicológicas de ser reconocidas como ciencia que muchos psicólogos se resisten a admitir el psicoanálisis como una escuela que hace contribuciones sobre su objeto de estudio. Los psicoanalistas, por su parte, basados en las críticas de Lacan a la ciencia, han pretendido distanciarse de la psicología porque asumen que psicología es exclusivamente aquella que emprendió un proyecto científico, desconociendo la diversidad que existe al interior de la disciplina y que no todas las corrientes psicológicas se embarcaron en el proyecto de devenir una ciencia. Hay una vertiente ascética en la psicología que, aunque recurre a procedimientos científicos, su principal interés no es la construcción de conocimientos validados por la ciencia, sino la transformación subjetiva. 
Consideramos entonces que el psicoanálisis hace parte de esta corriente ascética de la psicología.

4. El método experimental, también llamado hipotético-deductivo o galileanonewtoniano, no es el único método científico. Lo que caracteriza al método científico es la contrastación dialéctica entre la teoría y la práctica, y la experimentación es sólo una forma de llevar a cabo dicha contrastación, por lo tanto, hay otros métodos, como el clínico y el psicoanalítico, que también son científicos, que bien pueden aplicarse con el propósito de investigar un fenómeno y generar conocimiento o con el propósito de llevar a cabo un proceso de ascesis subjetiva, un tratamiento del alma.

5. Reconocer ambas corrientes en la psicologia (ascética y epistémica), concebir el método científico más allá de la experimentación y reafirmar el psicoanálisis como una escuela psicológica, tiene unas implicaciones que consideramos deseables, ya que es una forma de concebir la psicología, el psicoanálisis y la ciencia que va en contravía del espíritu dogmático de quienes promulgan su propia corriente y sus ideales como los únicos o verdaderos, alejándose así del espíritu pluralista, escéptico y receptivo que caracteriza a los científicos (Sagan, 1987), siempre dispuestos a volver a interrogar sus presupuestos y a estar atentos a los valores, emociones o al narcisismo, que pueden operar como sesgos o prejuicios en la labor científica y hacer que se prefiera una posición en lugar de otra. Las mutuas exclusiones entre psicoanalistas y psicólogos parecen basarse más en un modo de proceder sectario y dogmático que en argumentos epistemológicos.

6. Excluir de la psicología la tradición ascética en el abordaje del alma, más que una ganancia, representa un empobrecimiento de la disciplina, ya que una mirada de ambas tradiciones, sin prejuicios ni dogmas, permite reconocer que en muchos aspectos pueden complementarse, no excluirse. 


\section{Referencias}

Abbagnano, N. (1974). Diccionario de Filosofía (2a ed.). México: Fondo de Cultura Económica.

Aristóteles. (1983). Acerca del alma. Madrid: Gredos.

Bacon, F. (1984). Novum organum. Barcelona: Orbis.

Braunstein, N. (1979). Psicología, ideología y ciencia (6a ed.). México: Siglo XXI.

Cassirer, E. (1981). Filosofía de la Ilustración (2a ed.). México: Fondo de Cultura Económica.

Descartes, R. (1970). Discurso del método (12a ed.). Madrid: Espasa-Calpe.

Dilthey, W. (1945). Psicología y teoría del conocimiento. México: Fondo de Cultura Económica.

Foucault, M. (2006). Hermeneutica del sujeto. México: Fondo de Cultura Económica.

Freud, S. (1916). Conferencias de introducción al psicoanálisis (1915-1916). En Obras completas. Tomo XV (p. 219). Buenos Aires: Amorrortu Editores.

Freud, S. (1923a). Breve informe sobre el psicoanálisis (1924 [1923]). En Obras completas. Tomo XIX (pp. 199-221). Buenos Aires: Amorrortu Editores.

Freud, S. (1923b). Dos artículos de enciclopedia: "Psicoanálisis" y "Teoría de la libido" (1923 [1922]). En Obras completas. Tomo XVIII (pp. 228-354). Buenos Aires: Amorrortu Editores.

Freud, S. (1927). El porvenir de una ilusión (1927). En Obras completas. Tomo XXI (pp. 1-55). Buenos Aires: Amorrortu Editores.

Freud, S. (1932). 35 conferencia. En torno de una cosmovisión (1933 [1932]). En Obras completas. Tomo XXII (pp. 146-168). Buenos Aires: Amorrortu Editores.

Ginzburg, C. (1989). Morelli, Freud y Sherlock Holmes: indicios y método científico. En U. Eco \& T. Sebeok (Eds.), El signo de los tres. Barcelona: Lumen.

Lacan, J. (1982). El seminario de Jacques Lacan. Libro 20: Aún. Buenos Aires: Paidós. 
Lacan, J. (1983). El seminario de Jacques Lacan. Libro 2: El yo en la teoría de Freud y en la técnica psicoanalitica. Buenos Aires: Paidós.

Lopera, J. D. (2006). Psicología ascética y psicología epistémica. Acta Colombiana De Psicologia, 9, 75-86.

Lopera, J. D., Manrique, H., Zuluaga, M., \& Ortiz, J. (2010). El objeto de la psicología: el alma como cultura encarnada. Medellín: Editorial Universidad de Antioquia.

Nussbaum, M. (1995). El discernimiento de la percepción: una concepción aristotélica de la racionalidad privada y pública. Estudios de Filosofía (Medellin), (11).

Platón. (1998). Protágoras. Barcelona: Alianza Editorial.

Platón. (2005). Fedón. En Diálogos (29a ed., p. 605). México: Editorial Porrúa.

Platón. (2010). Apología de Sócrates. Barcelona: Editorial Gredos.

Ramírez, C. A. (1991). El método científico en el psicoanálisis. Revista Universidad de Antioquia, 60(224), 35-41.

Ramírez, C. A. (2012). La vida como un juego existencial: Ensayitos. Medellín: Fondo Editorial Universidad EAFIT.

Ramírez, C. A., Lopera, J. D., Zuluaga, M., Ramírez, V. E., Henao, C. M., \& Carmona, D. M. (2014). Relaciones psicología-psicoanálisis (2a ed.). Bogotá: San Pablo.

Sagan, C. (1987). La carga del escepticismo. Skeptical Inquirer, 12.

Watson, J. (1982). La psicología tal como la ve el conductista. En J. M. Gondra (Ed.), La psicología moderna. Textos básicos para su génesis y desarrollo (pp. 399-414). Bilbao: Desclée de Brouwer.

Wundt, W. (1982). Objeto, divisiones y método de la psicología. En J. M. Gondra (Ed.), La psicología moderna. Textos básicos para su génesis y desarrollo (pp. 181-196). Bilbao: Desclée de Brouwer.

Zambrano, M. (1987). El pensamiento vivo de Séneca. Madrid: Cátedra. 